\title{
Desigualdades socioeconômicas associadas ao excesso de peso e sedentarismo em adolescentes brasileiros
}

\author{
Socioeconomic inequalities in the prevalence of excess weight \\ and sedentary behavior among Brazilian adolescents
}

Carolina Souza Ferreira (https://orcid.org/0000-0002-7600-6690) ${ }^{1}$

Fabíola Bof de Andrade (https://orcid.org/0000-0002-3467-3989) ${ }^{1}$

${ }^{1}$ Programa de PósGraduação em Saúde Coletiva, Centro de Pesquisas René Rachou, Fundação Oswaldo Cruz (Fiocruz). Av. Augusto de Lima 1715, Barro Preto. 30190-002 Belo Horizonte MG Brasil.

fabiola.bof@fiocruz.br

\begin{abstract}
The scope of this study was to estimate the prevalence of excess weight and a sedentary lifestyle among Brazilian students between 13 and 17 years of age and investigate the magnitude of the socioeconomic inequalities related to these outcomes. A cross-sectional study was carried out based on data from the National School Health Survey (PeNSE) conducted in 2015. Inequalities were evaluated using the Absolute and Relative Index of Inequality. The prevalence of excess weight in Brazil was 24.2\%, ranging from 20.7\% in the Northeast Region to $27.8 \%$ in the Southern Region. The prevalence of a sedentary lifestyle in Brazil was $67.8 \%$, ranging from $61.8 \%$ in the North Region to $70.3 \%$ in the Southeast Region. The direct and positive association between better socioeconomic conditions and the prevalence of excess weight and a sedentary lifestyle indicate health inequities in Brazil. The results of this study point to the need to restructure and reinforce public policies directed at adolescents, which should have as guidelines the promotion of a healthy lifestyle and the reduction of health inequities.
\end{abstract}

Key words Obesity, Pediatric obesity, Adolescent, Socioeconomic factors, Health status disparities
Resumo O objetivo deste estudo foi estimar a prevalência de excesso de peso e sedentarismo em escolares brasileiros na faixa etária de 13 a 17 anos, e investigar a magnitude das desigualdades socioeconômicas relacionadas a esses desfechos. Realizou-se um estudo transversal com base nos dados da Pesquisa Nacional de Saúde do Escolar (PeNSE) do ano 2015. As desigualdades foram avaliadas por meio dos Índices Absoluto e Relativo de Desigualdade. A prevalência de excesso de peso no Brasil foi $24,2 \%$, variou de $20,7 \%$ na região Nordeste a 27,8\% na região Sul. Já a prevalência de sedentarismo no Brasil foi 67,8\%, variou de $61,8 \%$ na região Norte a $70,3 \%$ na região Sudeste. A associação direta e positiva entre melhores condições socioeconômicas e as prevalências de excesso de peso e sedentarismo indicam iniquidades em saúde existentes no Brasil. Os resultados do presente estudo apontam a necessidade de reestruturação e fortalecimento das políticas públicas voltadas aos adolescentes, que devem ter como diretrizes a promoção de estilos de vida saudáveis e a redução das iniquidades em saúde.

Palavras-chave Obesidade, Obesidade pediátrica, Adolescente, Fatores socioeconômicos, Disparidades nos niveis de saúde 


\section{Introdução}

O excesso de peso é considerado um dos mais sérios desafios da saúde pública no século XXI, devido ao rápido aumento nas prevalências e as graves consequências para a saúde dos indivíduos $^{1,2}$. Nas últimas três décadas a prevalência de excesso de peso aumentou consideravelmente em todas as faixas etárias no mundo ${ }^{3}$, chamando atenção para o significativo aumento entre as crianças e os adolescentes ${ }^{4}$. Entre 1980 a 2013 nos países desenvolvidos, o excesso de peso em crianças e adolescentes de 2 a 19 anos, aumentou de $16,9 \%$ para $23,8 \%$ entre meninos e de $16,2 \%$ para $22,6 \%$ entre meninas. Nos países em desenvolvimento, as prevalências eram de $8,1 \%$ e $8,4 \%$ entre meninos e meninas respectivamente em 1980 e aumentaram para $12,9 \%$ e $13,4 \%{ }^{5}$. Nessas faixas de idade o excesso de peso pode afetar a saúde, a educação e a qualidade de vida ${ }^{3}$. Adolescentes com excesso de peso apresentam maior risco de serem adultos obesos ${ }^{6}$ e de desenvolverem doenças crônicas ${ }^{3}$, tais como as doenças cardiovasculares e o diabetes ${ }^{7}$.

O excesso de peso é causado pelo acúmulo de gordura corporal, sob a forma de tecido adiposo e possui etiologia multifatorial, envolvendo fatores ambientais e genéticos ${ }^{8}$. Entre os fatores ambientais os achados dos estudos são consistentes em relação a associação positiva entre o excesso de peso, o consumo de alimentos não saudáveis e o sedentarismo ${ }^{3,9}$. Além disso, diferentes estudos têm demonstrado a existência de desigualdades socioeconômicas em relação ao excesso de peso ${ }^{10-}$ ${ }^{12}$. No entanto, a direção dessa associação varia de acordo com o nível de desenvolvimento socioeconômico do país. Estudos realizados em países ricos demonstram que o excesso de peso em adolescentes é mais prevalente entre indivíduos com piores condições socioeconômicas ${ }^{13-15}$, enquanto que nos países em desenvolvimento ocorre o inverso $^{16-18}$. As medidas socioeconômicas avaliadas por esses estudos incluíram escolaridade dos pais $^{13,14,16,17}$, renda familiar ${ }^{15,17}$ e ocupação dos pais ${ }^{16}$. No Brasil, poucos estudos de abrangência nacional avaliaram o excesso de peso entre adolescentes ${ }^{19}$. Estudos prévios, realizados no país, verificaram diferenças entre o excesso de peso e a condição socioeconômica a partir de análises bivariadas e sugeriram uma associação direta conforme observado nos países em desenvolvimento ${ }^{20-23}$. Porém, esses estudos não avaliaram a magnitude dessas diferenças utilizando medidas específicas para mensurar essas desigualdades.

Estudo recente demonstrou que entre países de alta e média/baixa renda as desigualdades socioeconômicas relacionadas ao comportamento sedentário foram melhores preditores da obesidade $^{24}$. No entanto, apesar do seu reconhecido papel como fator de risco modificável para o combate ao excesso de peso ${ }^{25,26}$, pouco se sabe sobre a sua prevalência entre os diferentes níveis socioeconômicos, apesar de estudos apontarem variações importantes em diferentes países ${ }^{3,12,27}$. Os resultados relativos às desigualdades no sedentarismo também são divergentes dependendo da forma como o sedentarismo foi avaliado ${ }^{27}$.

O limitado número de estudos incluindo a população de adolescentes dificulta a investigação dessas desigualdades e a avaliação do impacto das políticas públicas para a redução do excesso de peso entre os grupos mais vulneráveis. Assim, em consonância com os objetivos da Pesquisa Nacional de Saúde do Escolar (PeNSE) que estabelece a necessidade de se monitorar os fatores de risco para à saúde dos escolares brasileiros e identificar questões prioritárias para o desenvolvimento de política $^{28}$; o objetivo deste estudo foi investigar e quantificar a magnitude das desigualdades socioeconômicas relacionadas ao excesso de peso e ao comportamento sedentário entre adolescentes brasileiros.

\section{Métodos}

Foi realizado um estudo transversal com dados da última PeNSE realizada em 2015. Neste estudo utilizou-se a amostra dois da PeNSE 2015, que continha informações sobre peso e estatura, necessárias para avaliação do excesso de peso, totalizando uma amostra de 8.517 adolescentes para a análise das desigualdades no excesso de peso e 8.499 adolescentes para análise das desigualdades no comportamento sedentário. Essa amostra foi planejada para ser representativa do país e das grandes regiões para os escolares na faixa etária de 13 a 17 anos. Foram entrevistados estudantes do $6^{\circ}$ ao $9^{\circ}$ ano do ensino fundamental e da $1^{\text {a }}$ a $3^{\text {a }}$ série do ensino médio, devidamente matriculados no ano letivo de 2015, e frequentando regularmente escolas públicas e privadas situadas nas zonas urbanas e rurais de todo o território nacional com, no mínimo, 15 escolares matriculados nas séries escolhidas. Todos os detalhes da pesquisa e processo de amostragem foram publicados previamente ${ }^{28,29}$. As variáveis dependentes foram o excesso de peso e o sedentarismo. Aquela foi avaliada por meio do Índice de Massa Corporal (IMC) [razão entre o peso $(\mathrm{kg})$ e o quadrado 
da estatura $\left(\mathrm{m}^{2}\right)$ ] para idade e sexo (IMC-para-idade), expresso em escore $\mathrm{z}$, de acordo com o proposto pela Organização Mundial de Saúde (OMS, 2007). Adolescentes com escore-z $\geq+1$ e $<2$ são classificados com sobrepeso, adolescentes com escore- $z+2$ são classificados com obesidade. $\mathrm{O}$ excesso de peso abrange o sobrepeso e a obesidade, ou seja, escore- $z \geq+1^{30}$. A pesagem foi realizada com balança eletrônica portátil e o peso registrado em quilogramas, considerando a primeira decimal apresentada no visor da balança. A estatura foi medida por meio de estadiômetro portátil, sendo registrada em centímetros, considerando a primeira casa decimal ${ }^{29}$.

O comportamento sedentário total foi avaliado por meio da seguinte pergunta: "Em um dia de semana comum, quanto tempo você fica sentado(a) assistindo televisão, usando computador, jogando videogame, conversando com amigos(as) ou fazendo outras atividades sentado(a)? (não contar sábado, domingo, feriados e o tempo sentado na escola)". Indivíduos que relataram comportamento sedentário maior que 2 horas por dia foram considerados sedentários ${ }^{31}$.

A escolaridade dos pais, e em particular a da mãe, é considerada um importante fator de proteção para a saúde de crianças e adolescentes ${ }^{29}$. A forte associação com as condições econômicas confere a essa variável a possibilidade de ser utilizada como um importante proxy das condições socioeconômicas $^{32,33}$, conforme vem sendo utilizado em diferentes estudos com a população de adolescentes $^{13,14,16,17,22}$. Essa variável foi avaliada segundo o autorrelato do estudante de acordo com as seguintes categorias: não estudou, fundamental incompleto, fundamental completo, ensino médio incompleto, ensino médio completo, ensino superior incompleto e ensino superior completo. Outras covariáveis foram incluídas como ajuste nos modelos múltiplos, a saber: sexo (masculino, feminino); idade (13-15 anos, 16-17 anos); tipo de escola (pública ou privada); situação da escola (urbana, rural); região do Brasil (Sul, Sudeste, Centro-Oeste, Norte e Nordeste).

Foi realizada análise descritiva seguida de análise bivariada. A análise da associação entre as variáveis dependentes (excesso de peso e sedentarismo) e as variáveis independentes categóricas foi testada por meio do teste qui-quadrado com correção de Rao-Scott, que leva em consideração os pesos amostrais ${ }^{34,35}$. As prevalências ajustadas de cada um dos desfechos no Brasil e em cada uma das macrorregiões foram estimadas pelo modelo múltiplo de Poisson. O modelo múltiplo para o desfecho excesso de peso foi ajustado pelas seguintes variáveis: idade, sexo, escolaridade da mãe, tipo de escola e região do país. O modelo múltiplo para o sedentarismo foi ajustado pelas mesmas variáveis incluindo a situação da escola (urbano, rural).

A avaliação das desigualdades socioeconômicas relacionadas ao excesso de peso e ao sedentarismo foi feita por meio dos índices absoluto e relativo de desigualdade, respectivamente, Slope Index of Inequality - Índice Absoluto de Desigualdade (SII) e Relative Index of Inequality - Índice Relativo de Desigualdade (RII), utilizando a escolaridade materna como medida de posição socioeconômica. Os índices SII e RII são obtidos pela regressão da variável de saúde (excesso de peso e sedentarismo) sobre um escore de posição relativa, obtido a partir da medida de posição socioeconômica. A obtenção do escore de escolaridade materna foi obtido a partir da ordenação da amostra de forma crescente, da pior ("não estudou": escore igual a zero) para a melhor situação de escolaridade materna ("ensino superior completo": escore igual a um). A cada grupo de escolaridade materna foi atribuído um valor que corresponde ao ponto médio da distribuição cumulativa da medida ${ }^{36}$. O SII é a diferença absoluta da prevalência de excesso de peso/sedentarismo entre aqueles de maior posição socioeconômica (escore 1) e aqueles de menor posição socioeconômica (escore 0). O RII é a razão de prevalência (desigualdade relativa) entre os grupos de maior e menor nível socioeconômico. Um valor de SII superior a zero e um valor de RII maior do que um indicam que a prevalência de excesso de peso/sedentarismo é significativamente maior no grupo de maior posição socioeconômica. SII e RII foram estimados empregando-se modelos lineares generalizados para dados binomiais ${ }^{37}$. Todas as análises foram feitas no programa Stata 14.0 (Stata Corporation, College Station, TX, EUA), utilizando-se o comando survey, que permite considerar a estrutura complexa do processo amostral.

O projeto da PeNSE foi aprovado pela Comissão Nacional de Ética em Pesquisa (Conep), do Conselho Nacional de Saúde (CNS). Todos os participantes assinaram o Termo de Consentimento Livre e Esclarecido no momento da entrevista $^{29}$.

\section{Resultados}

Do total de participantes do estudo, 24,2\% apresentaram excesso de peso e $67,8 \%$ eram sedentários. A maioria era do sexo masculino (50,5\%) e 
encontrava-se na faixa etária de 13 a 15 anos de idade $(57,7 \%)$. No que diz respeito à dependência administrativa da escola, $85 \%$ eram estudantes de escolas públicas. A Tabela 1 apresenta as características demográficas e socioeconômicas e a distribuição da amostra segundo a prevalência de excesso de peso e comportamento sedentário. As variáveis idade, escolaridade da mãe, tipo de escola e Região do País foram significativamente associadas ao excesso de peso. As variáveis escolaridade da mãe, tipo de escola, Região do País e situação da escola foram significativamente associadas ao sedentarismo (Tabela 1).

A Figura 1 apresenta as prevalências ajustadas de excesso de peso e sedentarismo para o Brasil e em cada uma das macrorregiões. A prevalência de excesso de peso variou de $20,7 \%$ na região Nordeste a $27,8 \%$ na região Sul. A prevalência de sedentarismo variou de $61,8 \%$ na região Norte a $70,3 \%$ na região Sudeste.

A Tabela 2 apresenta a magnitude das desigualdades socioeconômicas absolutas (SII) e relativas (RII) ao excesso de peso e ao sedentarismo. As desigualdades absolutas e relativas no excesso de peso foram significantes para o Brasil [SII=7,3, IC95\%: $(2,7 ; 11,8)$; RII=1,37, IC95\%: $(1,12 ; 1,66)]$. Quanto as grandes regiões, verificou-se desigualdades absolutas e relativas nas regiões Norte [SII=8,8, IC95\%: $(0,4$; 17,2); RII=1,53, IC95\%: $(1,02 ; 2,29)]$ e Sudeste [SII=10,8, IC95\%: $(2,5 ; 19,1)$; RII=1,58, IC95\%: $(1,10 ; 2,28)]$. Com relação ao sedentarismo, verificou-se que as desigualdades absolutas e relativas foram significativas para o Brasil e todas as grandes regiões, com exceção do Sudeste.

\section{Discussão}

O presente trabalho quantificou a magnitude das desigualdades socioeconômicas relacionadas ao excesso de peso e ao comportamento sedentário entre adolescentes brasileiros. Observaram-se significantes desigualdades socioeconômicas para ambos os desfechos no Brasil e a análise por grandes regiões demonstrou resultados heterogêneos.

A prevalência de comportamento sedentário foi maior do que a observada para excesso de peso, havendo diferenças nas prevalências desses dois desfechos entre as regiões brasileiras. A estimativa para o excesso de peso foi um pouco maior àquela encontrada entre adolescentes brasileiros em $2009(23 \%)^{22}$. Na América Latina, a prevalência de excesso de peso entre adolescentes de 12 a 19 anos variou de $16,6 \%$ a $35,8 \%{ }^{38}$.
Tabela 1. Análise descritiva e distribuição da amostra segundo a prevalência de excesso de peso e comportamento sedentário.

\begin{tabular}{|c|c|c|c|c|}
\hline & \multicolumn{2}{|c|}{$\begin{array}{c}\text { Excesso de } \\
\text { peso }\end{array}$} & \multicolumn{2}{|c|}{ Sedentarismo } \\
\hline & Total & Sim & Total & Sim \\
\hline & $\%$ & $\%$ & $\%$ & $\%$ \\
\hline \multicolumn{5}{|l|}{ Demográficas } \\
\hline Idade & & $* *$ & & \\
\hline $13-15$ & 57,7 & 26,1 & 57,7 & 67,9 \\
\hline $16-17$ & 42,3 & 21,6 & 42,3 & 67,6 \\
\hline \multicolumn{5}{|l|}{ Sexo } \\
\hline Masculino & 49,5 & 24,6 & 49,6 & 66,7 \\
\hline Feminino & 50,5 & 23,9 & 50,4 & 68,8 \\
\hline \multicolumn{5}{|l|}{ Socioeconômicas } \\
\hline Escolaridade da mãe & & * & & $* * *$ \\
\hline Não estudou & 6,4 & $18,6^{*}$ & 6,4 & 44,9 \\
\hline $\begin{array}{l}\text { Fundamental ou } 1^{\circ} \\
\text { grau incompleto }\end{array}$ & 26,2 & 22,8 & 26,2 & 64,2 \\
\hline $\begin{array}{l}\text { Fundamental ou } 1^{\circ} \\
\text { grau completo }\end{array}$ & 9,0 & 22,8 & 9,0 & 67,8 \\
\hline $\begin{array}{l}\text { Ensino médio ou } 2^{\circ} \\
\text { grau incompleto }\end{array}$ & 8,8 & 22,9 & 8,8 & 68,7 \\
\hline $\begin{array}{l}\text { Ensino médio ou } 2^{\circ} \\
\text { grau completo }\end{array}$ & 25,4 & 24,2 & 25,4 & 71,4 \\
\hline $\begin{array}{l}\text { Ensino superior } \\
\text { incompleto }\end{array}$ & 6,2 & 24,6 & 6,2 & 72,6 \\
\hline $\begin{array}{l}\text { Ensino superior } \\
\text { completo }\end{array}$ & 18,0 & 29,7 & 18,0 & 73,8 \\
\hline Tipo de escola & & $* *$ & & $* * *$ \\
\hline Privada & 15 & 28,4 & 15 & 75 \\
\hline Pública & 85 & 23,5 & 85 & 66,5 \\
\hline Região do país & & $* * *$ & & $* * *$ \\
\hline Norte & 9,4 & 21,6 & 9,4 & 61,1 \\
\hline Nordeste & 28,4 & 20,3 & 28,4 & 63,3 \\
\hline Sudeste & 40,7 & 26,2 & 40,7 & 71,7 \\
\hline Sul & 13,6 & 27,7 & 13,6 & 70,5 \\
\hline Centro-Oeste & 8,0 & 25,2 & 8,0 & 66,6 \\
\hline Situação da escola & & & & $* * *$ \\
\hline Urbana & 94,8 & 24,4 & 94,8 & 69,5 \\
\hline Rural & 5,2 & 21,5 & 5,2 & 36,7 \\
\hline
\end{tabular}

Com relação ao sedentarismo, a prevalência foi superior quando comparada com países euro- 


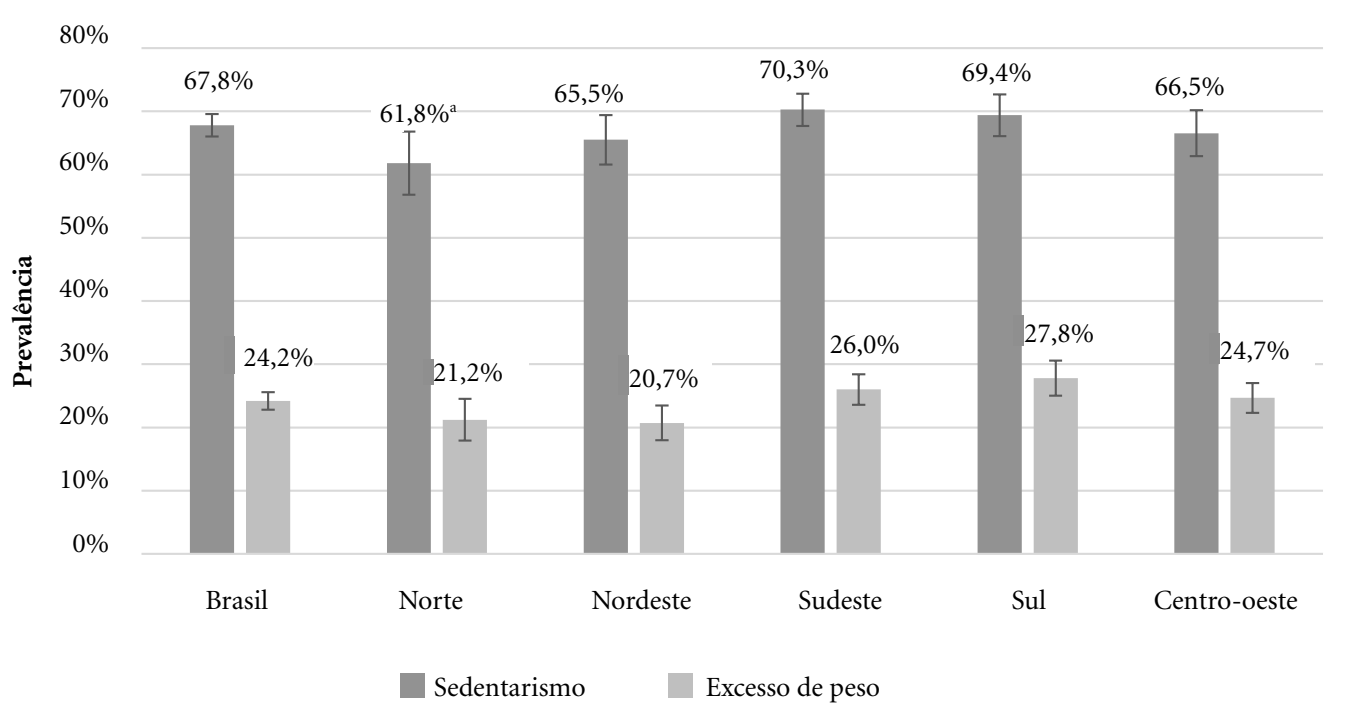

Figura 1. Prevalências ajustadas de excesso de peso e comportamento sedentário entre adolescentes no Brasil e grandes regiões.

Tabela 2. Desigualdade absoluta (SII) e relativa (RII) relacionada ao excesso de peso e sedentarismo entre adolescentes no Brasil e grandes regiões, de acordo com o indicador de escolaridade materna.

\begin{tabular}{|c|c|c|}
\hline & SII (95\%CI) & RII (95\%CI) \\
\hline \multicolumn{3}{|c|}{ Excesso de peso } \\
\hline Brasil & $7,3(2,7 ; 11,8)^{* *}$ & $1,37(1,12 ; 1,66)^{\star *}$ \\
\hline Norte & $8,8(0,4 ; 17,2)^{*}$ & $1,53(1,02 ; 2,29)^{*}$ \\
\hline Nordeste & $5,0(-4,4 ; 14,5)$ & $1,30(0,85 ; 2,01)$ \\
\hline Sudeste & $10,8(2,5 ; 19,1)^{\star}$ & $1,58(1,10 ; 2,28)^{\star}$ \\
\hline Sul & $2,8(-5,5 ; 11,1)$ & $1,10(0,82 ; 1,47)$ \\
\hline $\begin{array}{l}\text { Centro- } \\
\text { Oeste }\end{array}$ & $2,3(-5,0 ; 9,6)$ & $1,12(0,84 ; 1,50)$ \\
\hline \multicolumn{3}{|c|}{ Sedentarismo } \\
\hline Brasil & $14,7(9,5 ; 20,0)^{\star * *}$ & $1,21(1,12 ; 1,32)^{* * *}$ \\
\hline Norte & $25,2(13,6 ; 36,7)^{\star * *}$ & $1,49(1,18 ; 1,89)^{\star *}$ \\
\hline Nordeste & $26,5(15,8 ; 37,1)^{\star * *}$ & $1,48(1,24 ; 1,77)^{\star * *}$ \\
\hline Sudeste & $5,4(-4,4 ; 15,3)$ & $1,06(0,93 ; 1,22)$ \\
\hline Sul & $10,4(2,2 ; 18,6)^{*}$ & $1,15(1,03 ; 1,30)^{\star}$ \\
\hline $\begin{array}{l}\text { Centro- } \\
\text { Oeste }\end{array}$ & $16,0(7,2 ; 24,7)^{\star *}$ & $1,27(1,12 ; 1,45)^{\star *}$ \\
\hline
\end{tabular}

peus. Esse desfecho quando avaliado pelo tempo de televisão maior ou igual a duas horas por dia em países europeus variou entre $24 \%$ para meni- nas suíças a $71 \%$ para meninas ucranianas, sendo que a prevalência média considerando 39 países foi de $56 \%{ }^{12}$. Contudo, as diferenças na forma de avaliação precisam ser consideradas ao se comparar os estudos. Chaput et al..$^{39}$ observaram que iniquidades relativas ao tempo de tela foram maiores do que aquelas encontradas para outras medidas de sedentarismo (i.e., atividades físicas moderadas-vigorosas e tempo total de sedentarismo e tempo de sono), ressaltando que assim como a atividade física, políticas públicas baseadas na redução das desigualdades no tempo de tela devem ser incentivadas.

Quando comparados com estudos prévios, representativos da população de adolescentes brasileiros, os resultados mostram que a prevalência de excesso de peso ${ }^{20-22}$ e comportamentos sedentários ${ }^{40}$ são maiores nas regiões Sul, Sudeste e Centro-Oeste, que apresentam maior grau de desenvolvimento econômico, em comparação com as regiões Norte e Nordeste. Segundo Werneck et al. ${ }^{40}$, diferenças regionais no ambiente urbano e estrutura das cidades, incluindo a infraestrutura para a prática de atividade física, estariam entre os fatores contributivos para as desigualdades no comportamento sedentário entre as regiões do país.

A direção das diferenças de acordo com o nível de desenvolvimento econômico das regi- 
ões do país vai ao encontro das desigualdades observadas em relação ao grau de escolaridade das mães dos adolescentes. As razões pelas quais indivíduos de piores condições socioeconômicas têm menos prevalência de excesso de peso em países em desenvolvimento tem sido explicadas pela dificuldade para a obtenção de alimentos ${ }^{41}$ e o maior gasto calórico entre os mais pobres ${ }^{42}$. No Brasil, a ingestão de alguns alimentos de uma dieta saudável, como arroz, feijão, peixe fresco e farinha de mandioca, diminui à medida que a renda familiar per capita aumenta. Por outro lado, a ingestão de alguns alimentos de maior custo saudáveis (frutas, verduras e laticínios diet/light) e de alimentos não saudáveis (pizzas, salgados fritos, doces e refrigerantes) aumenta com o aumento da renda familiar ${ }^{43}$. Além disso, conforme observado neste e em outros estudos, no Brasil, indivíduos com pior condição socioeconômica apresentam menor prevalência de comportamento sedentário ${ }^{44,45}$ e maior prática de atividade física mensurada por meio do deslocamento ativo ${ }^{46}$. Por outro lado, nos países desenvolvidos, são observadas associações negativas entre condições socioeconômicas e os desfechos mensurados neste estudo, pois passaram por transições nutricionais e econômicas que favoreceram a adoção de estilos de vida mais saudáveis pela população com melhores condições socioeconômicas ${ }^{47}$. Apesar da associação positiva entre excesso de peso e condição socioeconômica observada nos países em desenvolvimento, em alguns desses países, incluindo o Brasil, a maior taxa de aumento na prevalência de excesso de peso tem sido observada entre os mais pobres ${ }^{48,49}$. No Brasil, o aumento da capacidade de compra das famílias de baixa renda, ampliou o acesso a alimentos ${ }^{50}$. Com isso, as famílias mais pobres atualmente têm maior chance de consumir algumas fontes mais baratas de calorias que, geralmente, são alimentos ultraprocessados com alta densidade energética, alto teor de gordura e açúcar, bem como com baixa qualidade nutricional (baixo teor de vitaminas e minerais) ${ }^{3,51,52}$.

Essas desigualdades a favor de indivíduos de melhor posição socioeconômica corroboram os achados de diferentes estudos realizados em países em desenvolvimento ${ }^{41,49}$, incluindo o Brasil ${ }^{20,22,23}$. Com relação ao comportamento sedentário, revisão sistemática recente ${ }^{53}$ demonstrou que a associação entre essa medida e as condições socioeconômicas é diferente entre países ricos e os de baixa-média renda, sendo que nestes a associação é positiva, conforme confirmado na presente análise. No entanto, os resultados encontrados para o
Brasil não são diretamente comparáveis a outros estudos uma vez que poucos avaliaram a magnitude das desigualdades para esses dois desfechos por meio do SII ou RII e empregaram a educação materna como posição socioeconômica.

Estudo realizado no Irã ${ }^{18}$ verificou uma desigualdade absoluta maior do que a encontrada neste estudo, sendo a prevalência de excesso de peso 10 pontos percentuais maior entre adolescentes pertencentes ao grupo de melhor condição socioeconômica, avaliada por meio de um escore de bens quando comparado ao grupo de menor status. Do ponto de vista relativo, metanálise realizada com estudos da região da África Subsariana ${ }^{16}$ confirmou a existência de desigualdades socioeconômicas a favor dos mais ricos. No entanto, diferente do encontrado no presente estudo a análise por subgrupos de acordo com a escolaridade parental não encontrou diferenças relativas entre os grupos (RII $=2,47,95 \% \mathrm{CI} 0,88$ 6,91 ). Porém a heterogeneidade dos estudos, as diferenças na mensuração da escolaridade parental, além do reduzido número de estudos podem ter influenciado os resultados. Estudo realizado com países Europeus e da América do Norte ${ }^{54}$ verificou que na maioria dos países havia desigualdades socioeconômicas relacionadas ao excesso de peso em adolescentes. Entre os dez países de renda-média que participaram do estudo, verificaram-se diferentes associações: quatro países apresentaram associação negativa entre a renda familiar e excesso de peso, três apresentaram associação positiva entre renda familiar e excesso de peso; e nos outros três houve associação negativa entre renda e excesso de peso para meninas e positiva para meninos. O SII foi maior do que o encontrado no presente estudo em dois países (Macedônia e Hungria) para meninos e em apenas um país para meninas (República Tcheca). Já o RII variou entre 0.52 para meninos da Macedônia a 4.80 para meninas da República Tcheca.

Este estudo é pioneiro em mensurar as desigualdades socioeconômicas na prevalência de excesso de peso e de sedentarismo dos adolescentes brasileiros em nível nacional e utilizando medidas complexas de avaliação das desigualdades. Essas medidas complexas apresentam em um único número a magnitude da desigualdade entre os subgrupos de pior e melhor posição socioeconômica, levando em consideração a distribuição populacional nos subgrupos intermediários. Por outro lado, a PeNSE, assim como outros inquéritos de base populacional, apresenta limitações: o uso do autorrelato da escolaridade materna pode ter enviesado a medida de posição socioeconô- 
mica, a depender do conhecimento do estudante acerca dessa informação. Além disso, por se tratar de um inquérito transversal, é necessária precaução na interpretação dos resultados, visto que não é possível estabelecer relação de causalidade para as associações encontradas.

Os resultados do presente estudo evidenciaram que as diferenças econômicas entre as grandes regiões brasileiras e as diferenças entre os níveis de escolaridade materna resultam em iniquidades em saúde. Atualmente, o Brasil passa por um momento de mudanças políticas e econômicas, com a sinalização de medidas para contenção de gastos públicos. Por isso, é necessá- rio que os governantes tenham cautela para que não haja redução de gastos em saúde que acarretem em regressão dos indicadores de saúde já conquistados e contribuam para o aumento das desigualdades em saúde entre os adolescentes, grupo este que carece de políticas públicas de saúde específicas. Assim, os achados desse estudo apontam para a necessidade de reestruturação e fortalecimento das políticas públicas já existentes que incluem o adolescente, tais como o Programa Nacional de Alimentação Escolar (PNAE) e o Programa Saúde na Escola (PSE), que devem ter como diretrizes a promoção de estilos de vida saudáveis e a redução das desigualdades.

\section{Colaboradores}

CS Ferreira e FB Andrade participaram do planejamento, análise de dados e redação do manuscrito. 


\section{Referências}

1. Brasil. Ministério da Saúde (MS). Cadernos da Atenção Básica: Obesidade. Brasília: MS; 2016.

2. World Health Organization (WHO). Global status report on noncommunicable diseases 2010. Geneva: WHO; 2011.

3. World Health Organization (WHO). Report of the commission on ending childhood obesity. Geneva: WHO; 2016.

4. Ncd Risk Factor Collaboration (NCD-RisC). Worldwide trends in body-mass index, underweight, overweight, and obesity from 1975 to 2016: a pooled analysis of 2416 population-based measurement studies in 128.9 million children, adolescents, and adults. Lancet 2017; 17:1-16.

5. Ng M, Fleming T, Robinson M, Thomson B, Graetz N, Margono C, Mullany EC, Biryukov S, Abbafati C, Abera SF, Abraham JP, Abu-Rmeileh NME, Achoki T, AlBuhairan FS, Alemu ZA, Alfonso R, Ali MK, Ali R, Guzman NA, Ammar W, Anwari P, Banerjee A, Barquera S, Basu S, Bennett DA, Bhutta Z, Blore J, Cabral N, Nonato IC, Chang J-C, Chowdhury R, Courville KJ, Criqui MH, Cundiff DK, Dabhadkar KC, Dandona L, Davis A, Dayama A, Dharmaratne SD, Ding EL, Durrani AM, Esteghamati A, Farzadfar F, Fay DFJ, Feigin VL, Flaxman A, Forouzanfar MH, Goto A, Green MA, Gupta R, Hafezi-Nejad N, Hankey GJ, Harewood HC, Havmoeller R, Hay S, Hernandez L, Husseini A, Idrisov BT, Ikeda N, Islami F, Jahangir E, Jassal SK, Jee SH, Jeffreys M, Jonas JB, Kabagambe EK, Khalifa SEAH, Kengne AP, Khader YS, Khang Y-H, Kim D, Kimokoti RW, Kinge JM, Kokubo Y, Kosen S, Kwan G, Lai T, Leinsalu M, Li Y, Liang X, Liu S, Logroscino G, Lotufo PA, Lu Y, Ma J, Mainoo NK, Mensah GA, Merriman TR, Mokdad AH, Moschandreas J, Naghavi M, Naheed A, Nand D, Narayan KMV, Nelson EL, Neuhouser ML, Nisar MI, Ohkubo T, Oti SO, Pedroza A, Prabhakaran D, Roy N, Sampson U, Seo H, Sepanlou SG, Shibuya K, Shiri R, Shiue I, Singh GM, Singh JA, Skirbekk V, Stapelberg NJC, Sturua L, Sykes BL, Tobias M, Tran BX, Trasande L, Toyoshima H, van de Vijver S, Vasankari TJ, Veerman JL, Velasquez-Melendez G, Vlassov VV, Vollset SE, Vos T, Wang C, Wang XR, Weiderpass E, Werdecker A, Wright JL, Yang YC, Yatsuya H, Yoon J, Yoon S-J, Zhao Y, Zhou M, Zhu S, Lopez AD, Murray CJL, Gakidou E. Global, regional, and national prevalence of overweight and obesity in children and adults during 1980-2013: a systematic analysis for the Global Burden of Disease Study 2013. Lancet 2014; 384(9945):766-781.

6. The NS, Suchindran C, North KE, Popkin BM, Gordon-Larsen P. Association of adolescent obesity with risk of severe obesity in adulthood. JAMA 2010; 304(18):2042-2047.

7. Us Preventive Services Task Force, Grossman DC, Bibbins-Domingo K, Curry SJ, Barry MJ, Davidson KW, Doubeni CA, Epling Jr JW, Kemper AR, Krist AH, Kurth AE, Landefeld CS, Mangione CM, Phipps MG, Silverstein M, Simon MA, Tseng C-W. Screening for Obesity in Children and Adolescents: US Preventive Services Task Force Recommendation Statement. JAMA 2017; 17(23):2417-2426.

8. World Health Organization (WHO). Population-based approaches to childhood obesity prevention. Genebra: WHO; 2012.
9. Enes CC, Slater B. Obesidade na adolescência e seus principais fatores determinantes. Rev Bras Epidemiol 2010; 13(1):163-171.

10. Cardoso LO, Engstrom EM, Leite IC, Castro IRR. Socioeconomic, demographic, environmental and behavioral factors associated with overweight in adolescents: a systematic literature review. Rev Bras Epidemiol 2009; 12(3):378-403.

11. Wang Y, Lim H. The global childhood obesity epidemic and the association between socio-economic status and childhood obesity. Int Rev Psychiatry 2012; 24(3):176-188.

12. Currie C, Zanotti CF, Morgan A, Currie DB, Looze M, Roberts C, editores. Social determinants of health and well-being among young people. Health Behaviour in School-aged Children (HBSC) study: international report from the 2009/2010 survey. Denmark: WHO Regional Office for Europe; 2012.

13. Smetanina $\mathrm{N}$, Albaviciute $\mathrm{E}$, Babinska V, Karinauskiene L, Albertsson-Wikland K, Petrauskiene A, Verkauskiene R. Prevalence of overweight/obesity in relation to dietary habits and lifestyle among 7-17 years old children and adolescents in Lithuania. BMC Public Health 2015; 15(1001):2-9.

14. Watts AW, Mason SM, Loth K, Larson N, Neumark -Sztainer D. Socioeconomic differences in overweight and weight-related behaviors across adolescence and young adulthood: 10-year longitudinal findings from Project EAT. Prev Med 2016; 87:194-199.

15. Rao DP, Kropac E, Do MT, Roberts KC, Jayaraman GC. Childhood overweight and obesity trends in Canada. Health Promot Chronic Dis Prev Can 2016; 36(9):194-198.

16. Fruhstorfer BH, Mousoulis C, Uthman OA, Robertson W. Socio-economic status and overweight or obesity among school-age children in sub-saharan africa - a systematic review. Clin Obes 2016; 6(1):19-32.

17. Desalew A, Mandesh A, Semahegn A. Childhood overweight, obesity and associated factors among primary school children in Dire Dawa, Eastern Ethiopia: a cross-sectional study. BMC Obes 2017; 4:20.

18. Kelishadi R, Qorbani M, Heshmat R, Djalalinia S, Sheidaei A, Safiri S, Hajizadeh N, Motlagh ME, Ardalan G, Asayesh H, Mansourian M. Socioeconomic inequality in childhood obesity and its determinants in Iran: a Blinder-Oaxaca decomposition. J Pediatr (Rio J) 2018; 94(2):131-139.

19. Simões CF, Lopes WA, Remor JM, Locateli JC, Lima FB, Santos TLC, Nardo Junior N. Prevalence of weight excess in Brazilian children and adolescents: a systematic review. Rev Bras Cineantropom Desempenho Hum 2018; 20(4):517-531.

20. Magalhães VC, Azevedo G, Mendonça S. Prevalência e fatores associados a sobrepeso e obesidade em adolescentes de 15 a 19 anos das regiões Nordeste e Sudeste do Brasil, 1996 a 1997. Cad Saúde Pública 2003; 19( Supl. 1):S129-S139.

21. Brasil. Ministério do Planejamento, Orçamento e Gestão. Instituto Brasileiro de Geografia e Estatística (IBGE). Diretoria de Pesquisas Coordenação de Trabalho e Rendimento Rio de Janeiro. Pesquisa de Orçamentos Familiares 2008-2009- Antropometria e Estado Nutricional de Crianças, Adolescentes e Adultos no Brasil. Brasília: IBGE; 2010. 
22. Araújo C, Toral N, Silva ACF, Velásquez-Melendez G, Dias AJR. Estado nutricional dos adolescentes e sua relação com variáveis sociodemográficas: Pesquisa Nacional de Saúde do Escolar (PeNSE), 2009. Cien Saude Colet 2010; 15(Supl. 2):3077-3084.

23. Leal VS, Lira PIC, Oliveira JS, Menezes RCE, Sequeira LAS, Arruda Neto MA, Andrade SLLS, Batista Filho M. Excesso de peso em crianças e adolescentes no Estado de Pernambuco, Brasil: prevalência e determinantes. Cad Saúde Pública 2012; 28(6):1175-1182.

24. Althoff T, Sosič R, Hicks JL, King AC, Delp SL, Leskovec J. Large-scale physical activity data reveal worldwide activity inequality. Nature 2017; 547(7663):336-339.

25. Organização Pan Americana da Saúde (OPAS). Plano de Ação para Prevenção da Obesidade em Crianças e Adolescentes. Washington: 66a Sessão do Comitê Regional da OMS; 2014.

26. Onis M. Preventing childhood overweight and obesity. J Pediatr (Rio J) 2015; 91(2):105-107.

27. Graf S, Cecchini M. Diet, physical activity and sedentary behaviours: Analysis of trends, inequalities and clustering in selected OECD countries. Paris: OECD Publishing; 2017.

28. Oliveira MM, Campos MO, Andreazzi MAR, Malta DC. Características da Pesquisa Nacional de Saúde do Escolar - PeNSE. Epidemiol Serv Saúde 2017; 26(3):605-616

29. Instituto Brasileiro de Geografia e Estatística (IBGE). Diretoria de Pesquisas, Coordenação de População e Indicadores Sociais. Pesquisa Nacional de Saúde do Escolar 2015. Rio de Janeiro: IBGE; 2016.

30. Onis M, Onyango AW, Borghi E, Siyam A, Nishida C, Siekmann J. Development of a WHO growth reference for school-aged children and adolescents. Bull World Health Organ 2007; 85:660-667.

31. Costa CS, Flores TR, Wendt A, Neves RG, Assunção MCF, Santos IS. Sedentary behavior and consumption of ultra-processed foods by Brazilian adolescents: Brazilian National School Health Survey (PeNSE), 2015. Cad Saúde Pública 2018; 34(3):e00021017.

32. Santos AMA, Jacinto PA, Tejada CAO. Causalidade entre renda e saúde: uma análise através da abordagem de dados em painel com os estados do Brasil. Estud Econ 2012; 42(2):229-261.

33. Barros AJD, Victora CG. Measuring Coverage in $\mathrm{MNCH}$ : Determining and Interpreting Inequalities in Coverage of Maternal, Newborn, and Child Health Interventions. PLoS Med 2013; 10(5):e1001390.

34. Rao JNK, Scott AJ. The analysis of categorical data from complex sample surveys: chi-squared tests for goodness of fit and independence in two-way tables. $J$ Am Stat Assoc 1981; 76:221-230.

35. Rao JNK, Scott AJ. On chi-squared for multiway contingency tables with cell proportions estimated from survey data. Ann Stat 1984; 12:46-60.

36. World Health Organization (WHO). Handbook on health inequality monitoring: with a special focus on low- and middle-income countries. Genebra: WHO; 2013.
37. Ernstsen L, Strand BH, Nilsen SM, Espnes GA, Krokstad S. Trends in absolute and relative educational inequalities in four modifiable ischaemic heart disease risk factors: repeated cross-sectional surveys from the Nord-Trøndelag Health Study (HUNT) 1984-2008. BMC Public Health 2012; 12:266.

38. Rivera JÁ, Cossío TG, Pedraza LS, Aburto TC, Sánchez TG, Martorell R. Childhood and adolescent overweight and obesity in Latin America: a systematic review. Lancet Diabetes Endocrinol 2014; 2(4):321-332.

39. Chaput, J-P, Barnes JD, Tremblay MS, Fogelholm M, Lambert EV, Maher C, Katzmarzyk PT. Inequality in physical activity, sedentary behavior, sleep duration, and risk of obesity in children: a 12-country study. Obesity Sci Practice 2018; 4(3):229-237.

40. Werneck AO, Oyeyemi AL, Fernandes RA, Romanzini M, Ronque ERV, Cyrino ES, Sardinha LB, Silva DR. Regional Socioeconomic Inequalities in Physical Activity and Sedentary Behavior Among Brazilian Adolescents. J Phys Act Health 2018; 15(5):338-344.

41. Subramanian SV, Perkins JM, Ozaltin E, Davey SG Weight of nations: a socioeconomic analysis of women in low- to middle-income countries. Am J Clin Nutr 2011; 93(2):413-421.

42. Sobal J, Stunkard AJ. Socioeconomic status and obesity: a review of the literature. Psychological Bull 1989; 105:260-275.

43. Instituto Brasileiro de Geografia e Estatística (IBGE). Coordenação de Trabalho e Rendimento. Pesquisa de orçamentos familiares 2008-2009: análise do consumo alimentar pessoal no Brasil. Rio de Janeiro: IBGE; 2011.

44. Hallal PC, Bertoldi AD, Gonçalves H, Victora CG. Prevalência de sedentarismo e fatores associados em adolescentes de 10-12 anos de idade. Cad Saúde Pública 2006; 22(6):1277-1287.

45. Dias PJP, Domingos IP, Ferreira MG, Muraro AP, Sichieri R, Gonçalves-Silva RMV. Prevalência e fatores associados aos comportamentos sedentários em adolescentes. Rev Saúde Pública 2014; 48 (2):266-274.

46. Ferreira RW, Varela AR, Monteiro LZ, Häfele CA, Santos SJ, Wendt A, Silva ICM. Desigualdades sociodemográficas na prática de atividade física de lazer e deslocamento ativo para a escola em adolescentes: Pesquisa Nacional de Saúde do Escolar (PeNSE 2009, 2012 e 2015). Cad Saúde Pública 2018; 34 (4):e00037917.

47. Pereira JL, Vieira DADS, Alves MCGP, César CLG, Goldbaum M, Fisberg RM. Excess body weight in the city of São Paulo: panorama from 2003 to 2015, associated factors and projection for the next years. $B M C$ Public Health 2018; 18(1):1332.

48. Conde WL, Monteiro CA. Nutrition transition and double burden of undernutrition and excess of weight in Brazil. Am J Clin Nutr 2014; 100(6):1617S-1622S.

49. Hernández-Cordero $S$, Cuevas-Nasu L, Morán-Ruán MC, Méndez-Gómez Humarán I, Ávila-Arcos MA, Rivera-Dommarco JA. Overweight and obesity in Mexican children and adolescents during the last 25 years. Nutr Diabetes 2017; 7(3):e247.

50. Brasil. Ministério da Saúde (MS). Secretaria de Atenção à Saúde. Departamento de Atenção Básica. Guia alimentar para a população brasileira. Brasília: MS; 2014. 
51. Drewnowski A. Obesity, diets, and social inequalities. Nutr Rev 2009; 67(Supl. 1):S36-S39.

52. Piryani S, Baral KP, Pradhan B, Poudyal AK, Piryani RM. Overweight and its associated risk factors among urban school adolescents in Nepal: a cross-sectional study. BMJ Open 2016; 6(5):e010335.

53. Mielke GI, Brown WJ, Nunes BP, Silva ICM, Hallal PC. Socioeconomic Correlates of Sedentary Behavior in Adolescents: Systematic Review and Meta-Analysis. Sports Med 2017; 47(1):61-75.

54. Due P, Damsgaard MT, Rasmussen M, Holstein BE, Wardle J, Merlo J, Currie C, Ahluwalia N, Sørensen TI, Lynch J; HBSC obesity writing group. Socioeconomic position, macroeconomic environment and overweight among adolescents in 35 countries. Int J Obes (Lond) 2009; 33(10):1084-1093.

Artigo apresentado em 23/01/2019

Aprovado em 03/05/2019

Versão final apresentada em 05/05/2019

Editores-chefes: Romeu Gomes, Antônio Augusto Moura da Silva 